\title{
CHIRURGISCHE GASTROENTEROLOGIE im Aufbruch
}

\author{
R. Bittner, Stuttgart
}

Der Beginn eines neuen Zeitabschnitts läßt uns auch heute noch einen Moment innehalten. Das Jahr 2000 und das 15jährige Jubiläum unserer Zeitschrift sind ein idealer Anlaß, um Bewährtes zu würdigen, aber auch um einen Aufbruch zum Neuen zu wagen. Bewährt hat sich, daß sich die CHIRURGISCHE GASTROENTEROLOGIE als erste Zeitschrift dieses Fachgebiets mit jedem Heft auf ein spezielles Thema konzentriert, dieses von verschiedenen Seiten beleuchtet und dabei ausgewiesene Experten zu Wort kommen läßt. Bewährt hat sich auch, daß sich die CHIRURGISCHE GASTROENTEROLOGIE interdisziplinär mit Gastroenterologie in Klinik, Praxis und Forschung befaßt. Sie ist mit diesem Konzept nach wie vor das einzige deutschsprachige Publikationsorgan. Auch zukünftig wird das interdisziplinäre Prinzip nicht nur beibehalten, sondern sogar noch stärker betont werden. Wir sehen hierin die herausragende Bedeutung unserer Zeitschrift, insbesondere auch unter Berücksichtigung der kontinuierlich abnehmenden finanziellen Ressourcen des Gesundheitssystems. Mehr denn je ist eine enge Zusammenarbeit der verschiedenen Disziplinen gefordert, vor allem auf den wichtigen Gebieten der Endoskopie und der Onkologie. Nur durch gemeinsame diagnostische und therapeutische Strategien sind die vorhandenen Mittel optimal zu nutzen. Unnötiges muß vermieden werden, um das Notwendige bezahlen zu können. Auch wenn tiefgreifende Rationalisierungen nicht zu umgehen sein werden, sollten Rationierungen nicht in Frage kommen. Um diesen zunehmenden Anforderungen gerecht $\mathrm{zu}$ werden, sind verschiedene inhaltliche und strukturelle Neuerungen in unserer Zeitschrift vorgesehen:

- Die Schriftleitung wird umstrukturiert in einen Hauptschriftleiter (Prof. Dr. R. Bittner, Stuttgart) sowie beigeordnet einen Schriftleiter Chirurgie (Prof. Dr. E. Klar, Heidelberg) und einen Schriftleiter Gastroenterologie (Prof. Dr. J. Mössner, Leipzig).

- Die wissenschaftlichen Beiräte werden entsprechend ihrer Arbeitsschwerpunkte einzelnen Spezialgebieten zugeordnet.

- Das Titelblatt wird in einem modernen Design neu gestaltet.

- Die bekannten und bewährten klinischen Schwerpunkte werden durch Sachgebiete wie Berufspolitik, Innovationen und Kongreßberichte erweitert. Es ist unser Anliegen, Sie auch über aktuelle Entwicklungen zu informieren, so daß Sie sich rechtzeitig auf neue Techniken einstellen oder mit neuem, hilfreichem Instrumentarium oder neuen Medizinprodukten vertraut machen können.

Das übergeordnete Ziel der CHIRURGISCHEN GASTROENTEROLOGIE ist traditionell, die interdisziplinäre Fort- und Weiterbildung auf höchstem Niveau zu fördern. Dies erreichen wir durch eine streng praxisnahe Auswahl von Themen, die von ausgewiesenen Spezialisten aus dem universitären, aber auch dem nichtuniversitären Bereich abgehandelt werden. Die Umgestaltung von Schriftleitung und wissenschaftlichem Beirat ist diesem Ziel untergeordnet, so daß die CHIRURGISCHE GASTROENTEROLOGIE noch aktueller und effektiver gestaltet werden kann. In diesem Kontext ist es uns auch ein Anliegen, die Zusammenarbeit mit unseren österreichischen und schweizerischen Kollegen zu intensivieren.

Inhaltlich wird die laparoskopische Chirurgie einen großen Raum einnehmen. Es hat für uns Priorität, Sie bei der rasanten Entwicklung dieses neuen und äußerst innovativen Zweigs der Viszeralchirurgie stets auf einem hohen Niveau zu informieren.

Für den klinischen Alltag haben Fragen der perioperativen Therapie herausragende Bedeutung. Auch hier sehen wir einen Schwerpunkt unserer Zeitschrift, die gerade dem jungen Kliniker Richtlinien in die Hand geben möchte, die es ihm erlauben, beispielsweise das sensible Feld der Ernährungs- und Antibiotikatherapie effektiv und kostengünstig zu gestalten.

Ich bin davon überzeugt, daß durch diese Veränderungen die Attraktivität der Zeitschrift nicht nur für den Chirurgen, sondern auch für den Gastroenterologen weiter zunehmen wird. Die CHIRURGISCHE GASTROENTEROLOGIE soll für jeden unverzichtbar sein, der durch einen interdisziplinären Ansatz in Diagnose und Therapie das Beste für seine Patienten tun möchte und dabei auch die aktuellen Kosten-Nutzen-Prinzipien im Auge behalten muß. Mit diesem einzigartigen Konzept versprechen wir, Sie umfassend und auch gemäß den Prinzipien einer «Evidencedbased Medicine» zu informieren, so daß Sie als Chirurg oder Gastroenterologe mit der CHIRURGISCHEN GASTROENTEROLOGIE eine kompetente Hilfe bei Ihren Entscheidungen in der täglichen Routine zur Hand haben.

\section{KARGER \\ Fax +497614520714 \\ (c) 2000 S. Karger GmbH, Freiburg \\ E-mail Information \\ Accessible online at: \\ www.karger.com}

